\title{
SADGAGE: A MCGDSS to Solve the Multicriteria Ranking Problem in a Distributed and Asynchronous Environment
}

\author{
Juan Carlos Leyva López ${ }^{1}$, Pável Anselmo Álvarez Carrillo ${ }^{1}$ \\ ${ }^{1}$ Universidad de Occidente, Culiacan, Mexico \\ juan.leyva@udo.mx, pavel.alvarez@udo.mx
}

\begin{abstract}
This paper presents a Web-based Multicriteria Group Decision Support System for solving multicriteria ranking problems: how to rank a set of alternatives - having evaluations in terms of several criteria - in decreasing order of preference by a collaborative group of decision makers in sequential or parallel coordination mode and in a distributed and asynchronous environment. The functional architecture incorporate the following features: The ELECTRE III model to aggregate multiple criteria preferences, a ELECTRE based method to aggregate the multiple criteria group preferences, an evolutionary algorithm to exploit a valued outranking relation, the Brainstorming technique to stimulate and to generate ideas, use of a facilitator tool for optimization the meetings coordination of the group members, use of an organize tool, use of a voting tool, use of a Graphic interface, a Group Norm subsystem, a Discussion subsystem and a Multiple Criteria Decision Analysis subsystem.
\end{abstract}

Keywords: Group Decision Support Systems, Multiple Criteria Decision Aid, Ranking Problem, Coordination Modes, ELECTRE Methods

\section{Introduction}

The strongest obstacle to resolving a group decision problem is that each individual has his/her own perception about problem. Consequently, he/she has his/her own belief about what should be the result or the correct decision to make. Therefore, in such an environment, it is logical and common to find conflicts between the opinion and desires of the group members. These conflicts arise due to the several factors present such as different values and objectives, different criteria and preference relations, lack of communication support between group members, etc. (Roy, 1996) encapsulates the diverse factors in conflict under the term "distinct value systems." Although these individual objectives are often strongly conflicting, a consensual decision must be reached.
In this paper, a Web-based Multicriteria Group Decision Support System (MCGDSS) called SADGAGE (Sistema de Apoyo a la Decisión en Grupo con Algoritmos Genéticos y Electre III on the World Wide Web) for solving ranking problems based on the ELECTRE-MOEA approach is presented. The SADGAGE system is built around a multicriteria outranking methodology for the ranking problem, which uses the ELECTRE III method (Roy, 1990), the ELECTRE based method to model group preferences (Leyva and Fernandez, 2003) and a multiobjective evolutionary algorithm (Leyva and Aguilera, 2005). The MCGDSS SADGAGE was designed to take place in distributed environments and to be conducted by a facilitator, where the group members can work in a dynamic environment (variation of time and space) in parallel or sequential coordination mode.

SADGAGE is a Web-based GDSS for Multicriteria Decision Analysis (MCDA). Being located on the WWW, it can be accessed from everywhere in the world. SADGAGE provides a common platform for collaborative group of decision makers that work in sequential or parallel coordination mode and in an asynchronous environment. The outranking model can be processed at the same or at different times and the results can be easily shared. The system SADGAGE is hosted in http://mcdss.udo.mx/xgdss/.

The structure of this paper is the following. In Section 2 we present the group multicriteria decision support system process. Related works are presented in Section 3. Section 4 presents the group MCDA processes with sequential and parallel coordination modes. Section 5 presents the systematization of the multicriteria group decision aiding process in a software tool as a MCGDSS prototype, and finally, Section 6 contains brief conclusions.

\section{Group MCDA Process}

Group MCDA processes usually require a facilitator to formulate initially the problem; moreover, successful group decision making requires appropriate coordination processes for incorporating diverse individual views into an aggregated final decision. Suitable decision support tools may facilitate the processes and help the group improve the decision quality (Malone and Crowston, 1990). 
In an asynchronous and distributed environment a key problem with the MCDA processes is the increased need for coordination of individual activities (Tindale, 1989). A coordination mode refers to a series of procedures and aggregation methods, which incorporate the group and individual members activities and facilitate them to reach agreement of a high quality group decision (Cao and Burstein, 2000). In such an environment, each participant can sometime work individually and/or collaborate with the rest of the group at other time.

We can distinguish two main general approaches, which use a multi criteria decision aid technique for aggregating group preferences:

A. In the first way, the group is asked to agree on the alternatives, criteria, scores, weights, thresholds and remaining parameters before the model provides a ranking. The group discussion focuses on what actions and criteria should be considered, what weights and other necessary parameters are appropriate. Once the discussion is closed and all the individual information has been gathered, a technique is used for obtaining values of these model parameters, which should represent the collective opinion. With this information, the multi criteria decision model gives us the group ranking.

B. Although members can exchange opinions and relevant information, a group consensus is needed only for defining the set of potential actions. Each member defines his own criteria, the appropriate evaluations and model parameters (weights, thresholds, etc.), and then the multi criteria decision aid method is used to get a personal ranking. Next, each actor is considered as a separate criterion, and the preferential information contained in its particular ranking is aggregated in a final collective ordering with the same (may be other) multi criteria decision approach (e.g. (Macharis et al., 1998), (Hwang and Lin, 1987), (Leyva and Fernandez, 2003)).

These two different processes result from two coordination modes, which Cao and Burstein (2000) have named sequential and parallel modes respectively.

\section{Related work}

There are a few GDSS that have used multiple criteria analysis techniques to support a group decision. Most of them have focused on communication elements, the structure of ideas, the generation of alternatives and the voting procedures. MCGDSS have emerged just in the 80 's, almost twenty years after the introduction of the field of MCDA. These methodologies were identified in Iz (1992), Iz and Gardiner (1993), and Hwang and Lin (1987). In the early years, Bui and Jarke (1986) and Bui (1987) present Co-oP, a co-operative multiple criteria group decision making system. The PLEXSYS system (Dennis et al., 1988) and its descendant GroupSystems (Nunamaker et al, 1991) contain, among others, a Alternative Evaluator tool which provide multiple criteria deci- sion making support. Carlsson et al. (1992) describe Alicia and Sebastian, a system for formalizing consensus reaching within a set of decision makers trying to find and agree upon a mutual decision. The system uses the Analytic Hierarchy Process (AHP) (Saaty, 1980) in order to model the preferences of each decision maker. After appears the Expert Choice system for group decision support based on the AHP method. Colson and Mareschal (1994) introduced JUDGES, a descriptive GDSS for the co-operative ranking of alternatives. WINGDSS (Csaki et al., 1995) is a group decision support software intended to aid one or more decision makers, from different fields but with a common interest, in ranking a predefined set of alternatives that are characterized by a finite set of criteria or attributes. Stanoulov (1995) presents the DIMCO method, an outranking approach forindividual and group decision making. This method is systematized on the UNIDAS 2. Also after Barzilai and Lootsma (1997) and Miettinen et al., (1997), describe some other interesting methods of multiple criteria group decision making support. Hamlainen and Mustajoki (1998) present the webHIPRE system, which, in part, is based on the AHP. In (Hamalainen et al, 1999), (Matsatsinis and Samaras, 2001) and (Davey and Olson, 1998) we can find another studies on MCGDSS. A MCGDSS based on the PROMETHEE approach was developed by Macharis et al. (1998).

Table 1 presents a summary by authors of the advances on the development of MCGDSS. The first column shows the author(s) and the second column shows the name of the system developed by them.

\begin{tabular}{ll} 
Table. 1: Advances in the development of MCGDSS. \\
\hline Author & MCGDSS \\
\hline Saaty (1980) & Team Expert Choice \\
& system with AHP \\
Bui and Jarke (1986) & Co-oP \\
Dennis et al. (1988) & PLEXSYS \\
Nunamaker et al. (1991) & GroupSystems \\
Carlsson et al. (1992) & Alicia and Sebastian \\
Colson and Mareschal (1994) & JUDGES \\
Csaki et al. (1995) & WINGDSS \\
Stanoulov (1995) & UNIDAS 2 \\
Hamlainen and Mustajoki & Web-Hipre System \\
(1998) & with AHP \\
Macharis et al. (1998) & PROMETHEE \\
Dias and Clímaco (2005) & VIP based GDSS \\
Shin, Shyur and Lee (2007) & TOPSIS based \\
& GDSS \\
Damart, Dias and Mousseau & IRIS with \\
(2007) & ELECTRE III \\
Xie et al. (2008) & VPRS based GDSS \\
Zhang (2009) & PSO-Fussy GDSS \\
Kacprzyk and Zadrozny & Web Intelligent \\
(2009) & \\
Ma, Lu and Zhang (2010) & Decider \\
Cebi and Kahraman (2010) & FIA based GDSS \\
\hline &
\end{tabular}




\section{Group MCDA Processes with Sequential and Par- allel Coordination Modes}

As stated above, we considered two coordination modes: parallel and sequential. We believe these two modes mostly cover the possible ways that people can go through a MCDA process. If the decision making process is guided by these two coordination modes, group may reach a consensus decision at a right time.

\subsection{Parallel Coordination}

Parallel coordination means everyone in a group works independently throughout most steps during the decision making process. The procedure and respective aggregation methods are described step by step as following:

Preliminary stage structuring the decision problem

The preliminary stage is a phase of knowledge acquisition and problem structuring. A facilitator has first to be appointed. On one hand, the facilitator has to be familiar with the GDSS-ELECTRE methodology and, on the other hand, he needs to have a reasonable knowledge of the actual group decision problem and its context. The following steps can be considered potentially.

Step1. First contact Facilitator - Decision Makers.

Each decision maker is encouraged to express his own opinions in order to progressively enrich the maturity of the facilitator with respect to the decision process.

Step2. Problem description.

The decision makers meet in the MCGDSS. The facilitator comments the available infrastructure and gives an overall description of the problem.

Step3. Define the possible evaluation criteria.

Step4. Alternative generation.

This is a "computer" phase during which the decision makers work alone.

Step5. Choose a stable set of alternatives.

This is a step were the decision makers reach a consensus decision on the final set of decision alternatives

Step6. Comments on the alternatives.

This step ends the preliminary stage and the next evaluation stages can start.

Individual evaluation stage

Step7. The proposed alternatives are evaluated by each criterion

Step8. Define weights and thresholds of the criteria

Step9. The individual ELECTRE III analysis is carried out by the facilitator

In this step, the ELECTRE III method (Roy, 1990) is applied to construct a valued outranking relation and next a evolutionary algorithm (Leyva and Aguilera, 2005) is applied to exploit it and as a result it recommends a complete ranking of the alternatives from the best to the worst ones.
During the individual evaluation stage, each decision maker works individually, with the possible assistance of the facilitator. At the end of this stage, everybody has a good personal view of the decision problem. Everybody has ideas on how to decide. More precisely, each decision maker has a ranking of the alternatives in decreasing order of preference.

Group evaluation stage.

The purpose is now to focus on group decision support in order to take into account the specific points by view of the different decision makers.

Step10. Global evaluation

At the end of the individual evaluation stage, the facilitator collects the rankings and valued preference relations coming from the decision makers and with these information the ELECTRE based method to model group preferences (Leyva and Fernandez, 2003) is applied to construct a valued outranking relation and again the evolutionary algorithm is applied to exploit it and as a result it recommend a complete ranking of the alternatives from the best to the worst ones.

At the end of step 10, a global evaluation is obtained for the group. The pair (ELECTRE based method to model group preferences-evolutionary algorithm) proposes a best compromise. If the group is agreeing upon the results of the global analysis, the best compromise can be adopted and the GDSS-ELECTRE session can be closed. On the other hand, if for some reasons some decision makers don't agree on this compromise, the conflicts have to be faced (See Figure 1 and Figure 2).

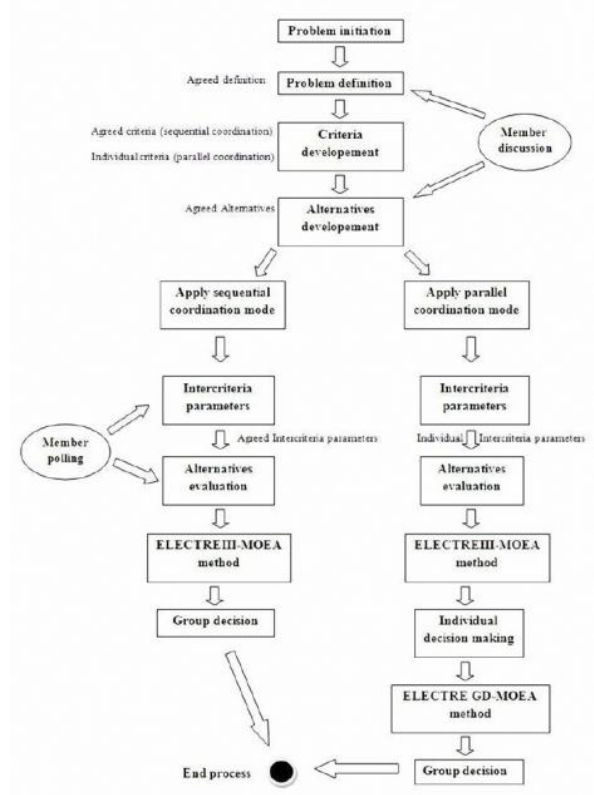

Fig. 1. Group MCDA process with two coordination modes 


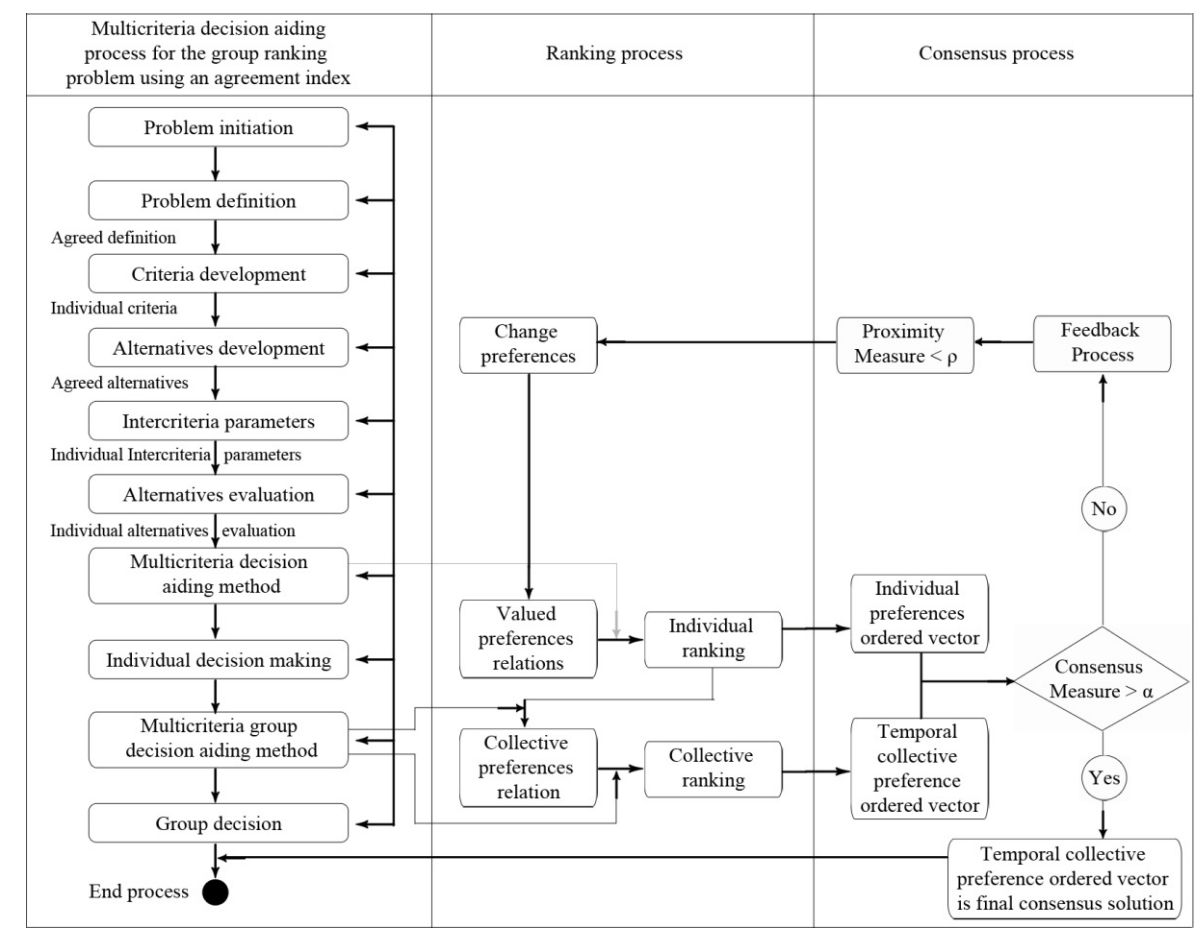

Fig. 2. Diagram of the GMCDA Consensus Process

\subsection{Sequential Coordination}

Sequential coordination implies that consensus would be sought throughout some stages of decision making process, from problem formulation to ranking determination. The consensus may be reached by applying aggregation methods at any appropriate step. The procedure with sequential coordination mode and ELECTRE IIIevolutionary algorithm methodology are not described by space limitations (see Figure 1).

\section{MCGDSS Prototype}

The MCGDSS helps collaborative groups to reach consensus in dynamic environmental meetings like distributed, asynchronous or synchronous. The use of these systems provides a structured framework to resolve multicriteria decision problems and to present results in a simple way. The MCGDSS provides a virtual space to develop electronic meetings. This allows the group to reach a common ground in the decision process and to apply systematized techniques through decision models. This kind of systems give participants a rational discussion model which allow to enhance the performance of the participants, the decision process quality and the definition of the meeting plan or agenda to reach the final solution of the problem.

The coordination modes functionality is the main contribution of the system, because the discussion model allows the facilitation and coordination of the decision process in two different working modes. On the one hand, the system provides the group members with a facility to work in an individual manner and generates a ranking for each individual, and later on it generates a collective consensus ranking. On the other hand, the decision process can be coordinated in a consensual manner, in which the members must agree in every stage of the process and therefore, obtain a collective ranking of alternatives.

\subsection{System Development Environment}

The prototype was developed in the Web platform and Microsoft.NET by using Visual Studio.Net Technology. The web site was developed with ASP.Net. The prototype is a groupware technology and consists of a combination of both software and services to transform the Internet into a personal medium for directing communication and interaction between some users, also provides an interactive environment for group collaboration, unencumbered by geography or time zones. The users directly interact with each other in a real-time environment and share in an asynchronous or synchronous manner.

\subsection{System Architecture}

The MCGDSS prototype was developed for the internet platform run within a web browser (See Figure 3). 


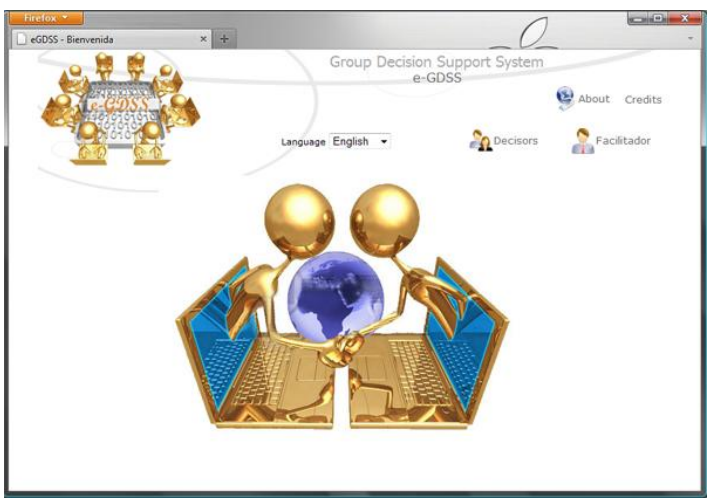

The Functional architecture incorporate the following features: The ELECTRE III model to aggregate multiple criteria preferences, the ELECTRE based model to aggregate the multiple criteria group preferences, an evolutionary algorithm to exploit a valued outranking relation, the Brainstorming technique to stimulate and to generate ideas, use of a facilitator tool for optimization the meetings coordination of the group members, use of an organize tool, use of a voting tool, use of a Graphic interface, a Group Norm subsystem, a Discussion subsystem and a Multiple Criteria Decision Analysis subsystem (See Figure 4).

Fig. 3. MCGDSS Prototype. Main window.

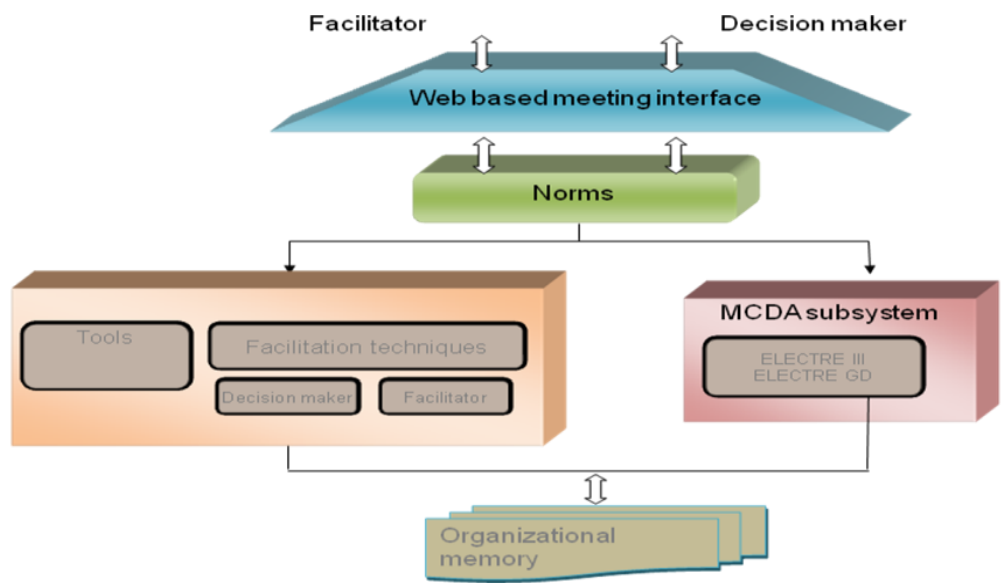

Fig. 4. MCGDSS Functional architecture.

Group Norm Subsystem. The Group Norm Subsystem has the goal of providing the MCGDSS with functions to explicitly support the definition and control of the decision making process to be adopted for the meeting. With this characteristic, more objectivity and shorter meetings are expected.

The system assumes the existence of two distinct types of actor, referred to as facilitator and group member. The facilitator is the person responsible for presenting the multicriteria decision problem, and defining the rules to be followed by participants during the meeting. The group members are the people that discuss, find possible courses of actions, define evaluation criteria and ranking the alternatives using multicriteria decision aid techniques. The person acting as facilitator can also plays the role of group member in the same meeting, but every meeting has one and only one facilitator.

The group norm is the central element of this subsystem, and it has the goal of documenting and controlling the rules defined for conducting the meeting. A meeting starts when the facilitator presents the description of the decision problem to the group and defining the group norm. Three main aspects are defined in the group norm: i) Participants of the meeting: enables the control of the integrity of the discussion and voting procedures. For example, only the facilitator may update the norm, and only people registered as a group member can discuss and vote.

ii) Discussion rules: define the duration of the discussion, whether the contributions are anonymous, and the number of interventions a group member can perform during the discussion. The definition of limits has the goal of achieving more objectivity in the meeting, by avoiding problems such as dominance. The facilitator must define convenient limits according to the characteristics of the decision problem and/or of the group, and must constantly monitor the process and update these limits whenever needed. The possibility of anonymity in the discussion seeks to reduce the problems related to hierarchical positions and interpersonal differences.

iii) voting rules: used to define how alternatives, criteria, weight, thresholds, etc. are extracted from the discussion, the number and the characteristics of each voting round, as well, by example, the alternatives to be considered in the next round in case no alternative gets the majority in the previous one. The properties defined for each round are its duration and the voting method. 
The MCGDSS provides functions supporting the invitation process, and the facilitator use it for that purpose. It is also assumed that every group member is well aware and accepts the rules defined for the meeting.

Discussion Subsystem. In this MCGDSS, the Kaner model was adopted, which will be briefly detailed. A problem may be divided in a set of more specific issues each one requiring a decision making process. Each process consists of one ore more zones. There are four different zones, which come in the following temporal order: divergent (search for information); groan (discuss issues); convergent (attempt to reduce the number of solutions); and closure (select one solution by consensus or voting). Each zone can consist of one or more strategies (patterns) for handling the issue. For instance, exploring the territory, searching for alternatives or discussing difficult issues are different strategies defined for the divergent zone. Finally, a strategy can consist of one or more activities. As an example, we find in the explore the territory strategy a sequence of activities characterized as who, what, when, where, and how (each activity identifies who is involved, what must be done and so forth). (See Figure 5).

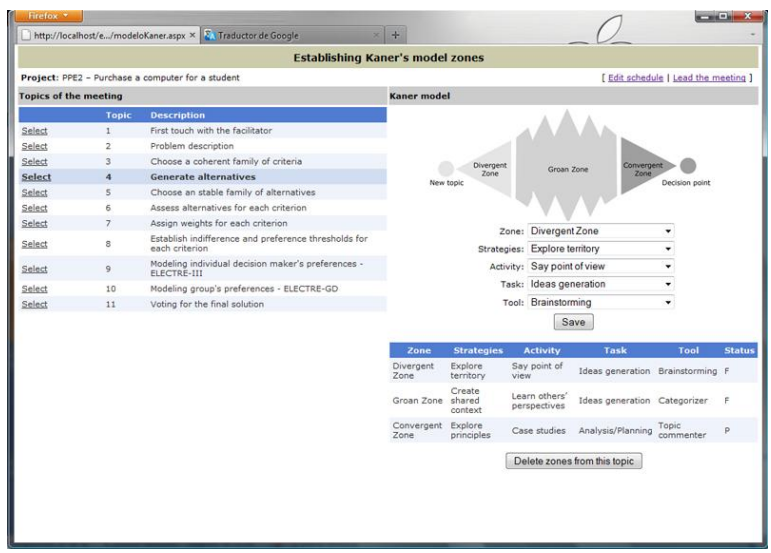

Fig. 5. The Kaner model windows.

The above model should be considered in a strict sense, as contingency descriptions of sub-processes.

Multiple Criteria Decision Analysis Subsystem. Following the sequential or parallel coordination modes, a consensus would be sought throughout some stages of decision making process, from problem formulation to ranking determination. The consensus may be reached by applying aggregation methods at any appropriate stage. A procedure with sequential coordination mode and ELECTRE III-evolutionary algorithm method is:

The group is asked to agree on the alternatives, criteria, weights, and thresholds before the model provides a ranking. The group discussion focuses on what actions and criteria should be considered, what weights and other necessary parameters are appropriate. Once the discussion is closed and all the individual information has been gathered, a technique is used for obtaining values of these model parameters, which should represent the collective opinion. With this information, the (ELECTRE III- evolutionary algorithm) method gives us the group ranking. It needs to be noticed that this procedure is iterative rather than simply sequential. If the group is unsatisfied with the result at any stage, it may go back to any step and redo it (See Figure 6).

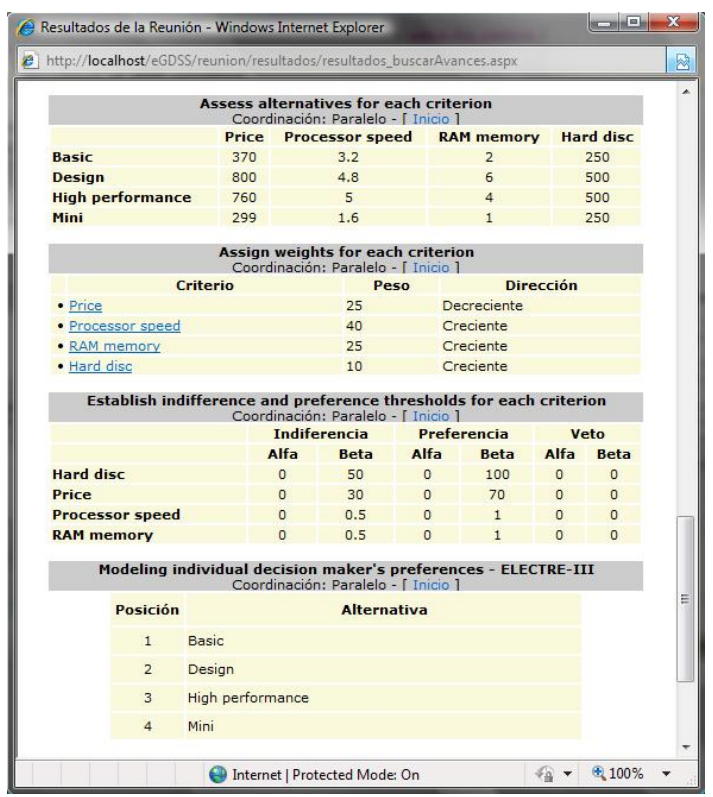

Fig. 6. MCDA windows.

\subsection{System Features}

The prototype provides support for group MCDA process at three levels:

a) Individual activity support

According to the Sequential and Parallel Coordination Mode, each group member can input the data/information group by group in dialogue boxes prompted by the system and contained in the Generate tool (Brainstorming). The grouped data/information is then stored in each participant's document, which can be accessed by the facilitator through the Facilitation tool. User's input of data/information may be organized and hierarchically displayed with the Organize tool and modified if needed. Participants can also assess the alternatives by calling embedded ELECTRE III model and feeding it in with their own decision preference. The ELECTRE III model component accepts weights and intercriteria parameter of the participant over each criterion. A rank of alternatives in decreasing order of preference based on participants' individual preferences is also displayed.

b) Group activity support

Within the sequential and parallel coordination mode, some steps need to reach a consensus between the group members. Once all participants' preferences are available, an aggregation of these preferences then takes place as a starting point for generating group decision preference afterwards. This aggregation information is distributed to each group member for polling. The polling result is regarded as group's aggregated preference if every partici- 
pant agrees, or another round of polling may be needed until a final consensus is reached.

c) Facilitation support

Facilitator plays an important role in the group decision making process supported by the system prototype. He or she controls process agenda and monitors process status with a facilitation tool. The facilitation based upon a predefined agenda determines the progression from one segment of decision making process to the next. The facilitation support component allows the facilitator to trace the participation status and progress of each group member (See Figure 7).

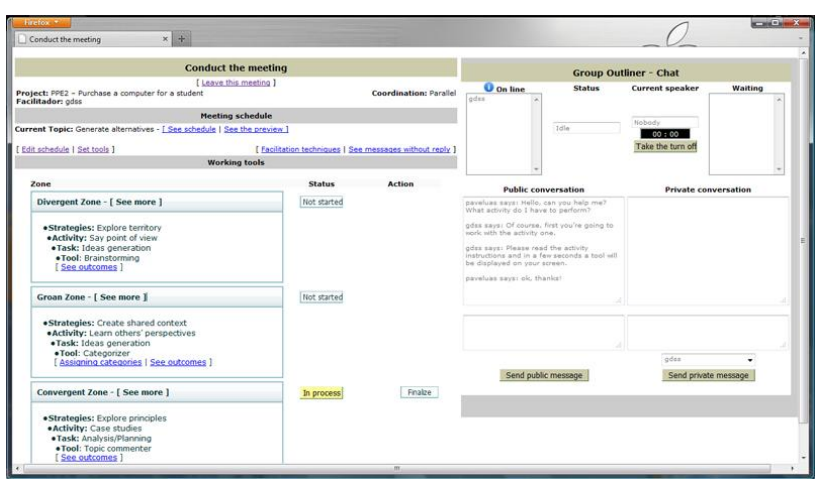

Fig. 7. Facilitation support. Driving the meeting.

\section{Conclusions}

In this paper, a MCGDSS to solve the ranking problem in a distributed and asynchronous environment and in a sequential or parallel coordination mode was presented. The striking feature of the proposed MCGDSS is the integrated use of the Kaner model, Multiple Criteria Decision Analysis techniques and an automated Group Norm, through a graphic interface that allows easy navigation across the subsystems using the web browser. These features aim at a) reducing the "noise" in the communication by introducing a systematic pattern for the discussion, b) facilitating the conveyance to a common solution, and c) allowing the definition of a meeting strategy, with mechanisms enabling decision-makers to stick to it.

The role of the facilitator in the MCGDSS is crucial. On one hand, the facilitator must be familiar with the ELECTRE III-evolutionary algorithm methodology, and on the other hand, he/she needs a good knowledge of the problem. The more the facilitator has prepared the session, the easier and the more efficient it will be.

\section{References}

[1] J. Barzilai, and F.A. Lootsma, "Power relations and group aggregation in the multiplicative AHP and SMART," Journal of multi-criteria decision analysis, 6, pp. 155-165, 1997.

[2] D. Bouyssou, "Building criteria: A prerequisite for MCDA.” In: C.A. Bana e Costa (ed.) Reading in mul- tiple criteria decision aid. Springer-Verlag, Berlin, pp. 58-80, 1990.

[3] T.X. Bui, “Co-oP: A group decision support system for cooperative multiple criteria group decision making," Lecture Notes in Computer Science, Vol. 290. Springer, Berlin, 1987.

[4] T.X. Bui and M. Jarque, "Communications designs for co-oP: A group decision support system," ACM Transactions on Office Information Systems, 4(2), pp. 81-103, 1986.

[5] P.P. Cao and F.V. Burstein, "An empirical study of influences of the coordination modes in supporting Group Multiple-Criteria Decision-Making," Australian Conference in Information Systems (ACIS'2000), Brisbane (CD ROM), 2000.

[6] C. Carlsson, D. Ehrenberg, P. Eklund, M. Fedrizzi, P. Gustafsson, P. Lindholm, G. Merkuryeva, T. Riisasen and A.G.S. Ventre, "Consensus in distributed soft environments," European Journal of Operational Research, 61, pp. 165-185, 1992.

[7] F.L. Cesar and J. Wainer, "vIBIS: A discussion and voting system," Journal of Brazilian Computer Society, 2 (1), pp. 36-43, 1994.

[8] G. Colson and B. Mareschal, "JUDGES: A descriptive group decision support system for the ranking of the items," Decision Support Systems, 12, pp. 391404, 1994.

[9] J. Conklin and M.L. Begeman "gIBIS: A hypertext tool for exploratory policy discussion," ACM Transactions on Office Information Systems, 6, pp. 303$331,1998$.

[10] P. Csaki, L. Csiszár, F. Folsz, K. Keller, C Mészáros, T. Rapcsák and P. Turchányi, “A flexible framework for group decision support WINGDSS Version 3.0," Annals of operations research, 58, pp. 441-453, 1995.

[11] A. Davey and D. Olson, "Multiple criteria decision making models in group decision support," Group Decision and Negotiation, 7, pp. 55-75, 1998.

[12] A.R. Dennis, J.F. George, L.M. Jessup, J.F. Nunamaker and D. Vogel, "Information technology to support electronic meetings," MIS Quarterly, 12 (4), pp. 591-624, 1998.

[13] R. P. Hamalainen and J. Mustajoki, "WebHIPRE-Java-applet for value tree and AHP analysis, computer software," http://www.hipre.hut.fi, Systems Analysis Laboratory, Helsinki University of Technology, 1998.

[14] R.P. Hamalainen, E. Kettunen, M. Marttunen and H. Ehtamo, "Towards decision and negotiation support in multi-stakeholder development of lake regulation policy," Proceedings of the $32^{\text {nd }}$ Hawaii International Conference on System Sciences, 1999.

[15] C. L. Hwang and M.J. Lin, "Group Decision Making under Multiple Criteria: Methods and Applications," Vol 281, Heildelberg, Germany: SpringerVerlag, 1987. 
[16] P.H. Iz, "Two multiple criteria group decision support systems based on mathematical programming and ranking methods," European Journal of Operational Research, 61 pp. 245-263, 1992.

[17] P.H. Iz and R.L. Gardiner, "Analysis of Multiple criteria decision support systems for cooperative groups," Group Decision and Negotiation, 2 (1), pp. 61-79, 1993.

[18] M.T. Jelassi, G. Kerstenand S. Zionts, "An introduction to group decision and negotiation support," In: C.A. Bana e Costa (Ed.): Readings in Multiple Criteria Decision Aid. Springer Berlin, 1990.

[19] J.C. Leyva and E. Fernandez Gonzalez, "A New Method for Group Decision Support Based on ELECTRE-III Methodology," European Journal of Operational Research, 148(1), pp. 14-27, 2003.

[20] J.C. Leyva and M.A. Aguilera, "A Multiobjective Evolutionary Algorithm for Deriving Final Ranking from a Fuzzy Outranking Relation," in C.A. Coello Coello, E. Zitzler and A. Hernández Aguirre (Editors), Evolutionary Multi-Criterion Optimization. Third International Conference, EMO 2005 , pp. 235-249, Springer, Lecture Notes in Computer Science, Vol. 3410, 235-249, 2005.

[21] C. Macharis, J.P. Brans and B. Mareschal, "The GDSS PROMETHEE Procedure," Journal of Decision Systems, 7, 1998.

[22] T.W. Malone and K. Crowston, "What is coordination theory and how can it help decision cooperative work systems?," In Proceedings of the ACM conference on computer-supported cooperative work, Los Angeles: The Association for Computer Machinery, pp. 357-370, 1990.
[23] N.F. Matsatsinis and A.P. Samaras, "MCDA and preference disaggregation in group decision support systems," European Journal of Operational Research, 130, pp. 414-429, 2001.

[24] K. Miettinen, P. Salminen and J. Hokkanen, "Acceptability analysis for multicriteria problems with multiple decision maker," Report 2/1997, University of Jyvaskyla, Department of mathematics, laboratory of scientific computing, Jyvaskyla, 1997.

[25] J.F. Nunamaker, A.R. Dennis, J.S. Valecich, D.R. Vogel and J.F George, "Electronic meeting systems to support group work," Communications of the ACM, 34 (7), pp. 40-60, 1991.

[26] B. Roy, "The outranking approach and the foundations of ELECTRE methods," In: C.A. Bana e Costa, (ed.) Reading in multiple criteria decision aid. Springer-Verlag, Berlin, pp. 155-183, 1990.

[27] B. Roy, "Multicriteria methodology for decision aiding," Nonconvex Optimization and its applications, Vol 12. Kluwer Academic Publishers, the Netherlands, 1996.

[28] T. Saaty, "The Analytic Hierarchy Process," McGraw Hill, New York, 1980.

[29] N. Stanoulov, "A parsimonious outranking method for individual and group decision making and its computerized support," IEEE Transactions on systems man and Cybernetics, 25, pp. 266-276, 1995.

[30] R.S. Tindale, "Group vs. Individual information processing: The effects of outcome feedback on decision making," Organizational Behavior and Human Decision Process, 44 pp. pp. 454-473, 1989. 